\title{
Histone deacetylase 2 is involved in $\mu$-opioid receptor suppression in the spinal dorsal horn in a rat model of chronic pancreatitis pain
}

\author{
YONG-HUI LIAO $^{1 *}$, JIAN WANG $^{1 *}$, YAN-YAN WEI $^{1}$, TING ZHANG $^{1}$, \\ YONG ZHANG $^{1}$, ZHONG-FU ZUO ${ }^{2}$, XIAO-YU TENG ${ }^{1}$ and YUN-QING LI ${ }^{1,3}$
}

\begin{abstract}
${ }^{1}$ Department of Anatomy and K.K. Leung Brain Research Centre, Preclinical School of Medicine, Fourth Military Medical University, Xi'an, Shaanxi 710032; ${ }^{2}$ Department of Anatomy, Histology and Embryology, Liaoning Medical University, Jinzhou, Liaoning 121000; ${ }^{3}$ Collaborative Innovation Center for Brain Science, Fudan University, Shanghai 200032, P.R. China
\end{abstract}

Received July 2, 2016; Accepted April 24, 2017

DOI: $10.3892 / \mathrm{mmr} .2017 .8245$

\begin{abstract}
Chronic pain occurs in $~ 85-90 \%$ of chronic pancreatitis (CP) patients. However, as the pathogenesis of $\mathrm{CP}$ pain remains to be fully understood, the current therapies for $\mathrm{CP}$ pain remain inadequate. Emerging evidence has suggested that the epigenetic modulations of genes are involved in chronic pain. In the present study, intrapancreatic trinitrobenzene sulfonic acid infusions were used to establish a CP model in rats. Mechanical allodynia was measured with von Frey filaments. Immunofluorescent staining analysis was used to observe the expression changes of histone deacetylase 2 (HDAC2) and $\mu$-opioid receptor (MOR), and intrathecal administration of the selective HDAC2 inhibitor AR-42 was used to assess the underlying mechanisms. The expression levels of c-Jun $\mathrm{N}$-terminal kinase $(\mathrm{JNK})$ in the thoracic spinal cord were detected by western blotting, and the mRNA expression levels of interleukin (IL) $1-\beta$, IL- 6 and tumor necrosis factor (TNF)- $\alpha$ were detected by reverse transcription-quantitative polymerase chain reaction. The results demonstrated that HDAC2 expression was upregulated during the course of $\mathrm{CP}$ induction, while MOR activity in the thoracic spinal dorsal horn was significantly suppressed. Intrathecal infusion of AR-42 significantly attenuated $\mathrm{CP}$-induced mechanical allodynia, with rescued MOR activity. Additionally, HDAC2 facilitated the release of inflammatory cytokines, including IL-1 $\beta$, IL- 6 and TNF- $\alpha$. These results suggested that the underlying mechanisms of
\end{abstract}

Correspondence to: Professor Yun-Qing Li, Department of Anatomy and K.K. Leung Brain Research Centre, Preclinical School of Medicine, Fourth Military Medical University, 169 Changle West Road, Xi'an, Shaanxi 710032, P.R. China

E-mail: deptanat@fmmu.edu.cn

*Contributed equally

Key words: chronic pancreatitis, histone deacetylase, epigenetic, $\mu$-opioid receptor, allodynia
HDAC2 regulating MOR activity under CP induction may occur via promoting the release of inflammatory cytokines, thus activating the JNK signaling pathway. The present study suggested that the epigenetic-regulated disturbance of MOR is dependent on the endogenous analgesia system in $\mathrm{CP}$, which may a provide novel therapeutic strategy for treating pain in CP.

\section{Introduction}

Chronic pancreatitis (CP) is a painful inflammatory disease, characterized by progressive destruction of the pancreatic gland and severe abdominal pain (1). Approximately 85-90\% $\mathrm{CP}$ patients suffer from abdominal pain (2). Pain in CP has been associated with malnutrition, narcotic addiction, and physical and emotional disability, which leads to major socioeconomic problems (3). However, the currently available therapies for $\mathrm{CP}$ pain remain inadequate and the underlying mechanisms remain to be completely elucidated. Previous reports have demonstrated that $\mathrm{CP}$-induced pain exhibits numerous characteristics similar to neuropathic pain, especially the alterations located in the central nervous system (CNS) (4). Epigenetic modulations of gene expression have been indicated to be involved in the development of chronic pain (5). Epigenetic alterations are required for long-lasting neuronal plasticity that is essential for the development of chronic pain state modifications $(6,7)$. Epigenetic modifications regulate the compaction of chromatin and include a variety of facets; significant epigenetic control is achieved via histone acetylation. Histone acetylation modification is dynamic and reversible, and is regulated by histone acetyltransferases (HATs) and histone deacetylases (HDACs) (8). Typically, HATs acetylate the histones to produce an open chromatin conformation, thus favoring gene expression, while HDACs deacetylate the DNA and result in a closed chromatin conformation and ultimately gene repression (9). Studies on HDACs inhibitors have demonstrated obvious analgesic effect on nociceptive responses of rodents, either delivered systemically or intrathecally (10-13). However, little is known on how this mechanism operates and which target genes are involved (14). Furthermore, whether selective 
interruption of HDAC activity on CP could alleviate allodynia remains to be investigated.

In the nervous system, activation of $\mu$-opiod receptor (MOR) leads to neuronal inhibition and causes an endogenous analgesia effect (15). The expression level of MOR in the mouse brain correlates with alterations in histone modifications (16). However, limited studies have analyzed the epigenetic processes that contribute to gene repression and activation in pain states. Furthermore, a study reported that MOR is mainly negatively regulated by the c-Jun NH2-terminal kinase (JNK) signaling pathway (17). Our previous study demonstrated that HDAC2 activity was significantly upregulated in the thoracic spinal cord, based on a rat CP model induced by intrapancreatic infusion of trinitrobenzene sulfonic acid (TNBS) (18), indicating that epigenetic regulation mechanisms are involved in chronic pain induced by $\mathrm{CP}$.

The present study aimed to investigate whether upregulation of HDAC2 affects MOR expression, and thus has an impact on CP allodynia. It was hypothesized that the elevated HDAC2 expression suppressed MOR activation via JNK signaling pathways, and aggravated CP pain. To test this hypothesis, AR-42 was used as a selective HDAC2 inhibitor (19), and the underlying mechanisms of $\mathrm{CP}$ pain were investigated.

\section{Materials and methods}

Animals. The present study was approved by the Animal Use and Care Committee for Research and Education of the Fourth Military Medical University (Xi'an, China), following the ethical guidelines on investigating experimental pain in conscious animals. 54 young adult male Sprague-Dawley rats (age, 10-12 weeks; weight, 180-220 g) were purchased from the Laboratory Animals Center (Fourth Military Medical University, Xi'an, China) and caged in a temperature-controlled environment at $22-25^{\circ} \mathrm{C}$ and $55 \pm 5 \%$ relative humidity with a 12-h light/dark cycle. Free access to water and food was available until $12 \mathrm{~h}$ before pancreatitis induction. Minimum animals were used for consistent effects.

Induction of pancreatitis and pain behavioral test. All rats were randomly divided into three main groups: TNBS $(n=30)$, sham $(n=18)$ and controls $(n=6)$. Rats in the TNBS and sham group were further divided for drug injection: TNBS-HDAC inhibitor (i)/saline and sham-HDACi/saline groups ( $n=6 /$ group). In order to study the time course of HDAC2 and MOR changes, 6 rats in the TNBS group were sacrificed at 1, 3 and 5 weeks each following TNBS infusion, in addition to 6 rats in the control and 6 rats in the sham group. All rats were sacrificed following anesthetization with pentobarbital (cat. no. 1507,002; Sigma-Aldrich; Merck KGaA; Darmstadt, Germany; 60 mg/kg, intraperitoneal injection) for further experiments 5 weeks after surgery. The protocol has been published in our previous study (18) with certain improvement for minimizing surgical injury. All the procedures in sham group were identical to the TNBS group, except the same volume of saline infusion. Mechanical allodynia was measured with von Frey filaments (Stoelting Co., Wood Dale, IL, USA) and performed fully randomized and blinded. The protocol was performed according to our previous study (18).
Intrathecal operation and drug administration. Intrathecal drug administration procedure was performed as previous described (18). The HDAC2-selective inhibitor AR-42 (AdooQ Bioscience, Irvine, CA, Canada) was used for the testing (19). The treatment group received an intrathecal injection of AR-42 (30 nmol) at each time point $(0,1,3$ and 5 weeks), while the same volume of saline was administered to the control group. Drug administration was performed under anesthetizing with isoflurane using a Vaporizer (Harvard Apparatus, Holliston, MA, USA), through an intrathecal catheter controlled by a micro-injection pump.

Immunofluorescent staining analysis. Rats were perfused through the ascending aorta with $100 \mathrm{ml} 0.9 \%$ saline after deeply anesthetizing with pentobarbital $(60 \mathrm{mg} / \mathrm{kg}$, intraperitoneally), following administration of $500 \mathrm{ml} 0.1 \mathrm{M}$ phosphate buffer ( $\mathrm{PB}, \mathrm{pH} 7.4$ ) containing $4 \%$ paraformaldehyde and $2 \%$ picric acid. The thoracic spinal segments T10-T12 were harvested and post-fixed with the same fixative for $4 \mathrm{~h}$, then cryosectioned at $4^{\circ} \mathrm{C}$ for $24 \mathrm{~h}$ in $0.1 \mathrm{M}$ PB containing $30 \%$ sucrose. Samples were transversely cut $(25-\mu \mathrm{m}$ thick) in a cryostat, then washed in PBS (0.01 M, pH 7.3) for 10 min three times. Sections were then blocked with $2 \%$ goat serum (EMD Millipore, Billerica, MA, USA) in $0.01 \mathrm{M}$ PBS containing $0.3 \%$ Triton $\mathrm{X}-100$ for $1 \mathrm{~h}$ at room temperature. These sections were incubated at $4^{\circ} \mathrm{C}$ overnight with the following primary antibodies: rabbit anti-HDAC2 (cat. no. sc-437285; 1:1,000; Santa-Cruz Biotechnology, Inc., Dallas, TX, USA) guinea pig anti-MOR (cat. no. AB5509; 1:200; EMD Millipore), mouse anti-neuronal-specific nuclear protein (NeuN; cat. no. MAB377; 1:500; EMD Millipore), mouse anti-glial fibrillary acidic protein (GFAP; cat. no. NE1015; 1:500; EMD Millipore) and mouse anti-integrin $\alpha-\mathrm{M}$ (CD11b) antibody (OX-42; cat. no. CBL1512; 1:500; EMD Millipore). The sections were then washed three times with PBS $(10 \mathrm{~min}$ each) and then incubated for $2 \mathrm{~h}$ at room temperature with the corresponding secondary antibody: Alexa 488-conjugated donkey anti-rabbit IgG (cat. no. R37118; 1:800; Molecular Probes; Thermo Fisher Scientific, Inc., Waltham, MA, USA) and Alexa 594-conjugated donkey anti-guinea pig IgG (cat. no. A-11076; 1:200; Molecular Probes; Thermo Fisher Scientific, Inc.). Images were obtained under a confocal laser microscope (FV1000; Olympus Corporation, Tokyo, Japan), and digital pictures were captured with the Fluoview 1000 imaging system (Olympus Corporation). A total of 12 nonadjacent sections were randomly selected for scanning. The $\mathrm{z}$-separation was $4.6 \mu \mathrm{m}$ under a $\mathrm{x} 20$ objective magnification, and was $1.0 \mu \mathrm{m}$ under a x60 objective magnification. For semiquantification, the fluorescent brightness value of immunoreactivities was detected on the same areas of the thoracic spinal dorsal horn using software (Fluoview; version 1.5.0.14; Olympus Corporation, Tokyo, Japan) under an Olympus IX-70 confocal microscope (Olympus Corporation).

Reverse transcription-quantitative polymerase chain reaction $(R T-q P C R)$. Rat thoracic spinal cord samples were harvested as described above. RNA extraction was performed using TRIzol (Gibco; Thermo Fisher Scientific, Inc.), and oligo(dT) primer and SuperScript II reverse transcriptase (Invitrogen; Thermo Fisher Scientific, Inc.) 
were used in cDNA RT. The mRNA expression levels of the pro-inflammatory cytokines interleukin (IL)-6, IL-1 $\beta$ and tumor necrosis factor (TNF)- $\alpha$ were assessed by RT-qPCR. The protocol was performed according to a previous report (18): $3 \mathrm{~min}$ at $9^{\circ} \mathrm{C}$, followed by 45 cycles of $10 \mathrm{sec}$ at $95^{\circ} \mathrm{C}$ for denaturation, and $45 \mathrm{sec}$ at $60^{\circ} \mathrm{C}$ for annealing and extension. All experiments were repeated twice, and PCR reactions were triplicate in each test. Target cDNA quantities were evaluated from the quantitation cycle number (Cq) (20) using Sequence Detection System software (version 2.4.1; Applied Biosystems; Thermo Fisher Scientific, Inc.). Primer sequences are provided in Table I. GAPDH served as an endogenous internal standard control.

Western blotting. Rats were sacrificed and T10-T12 spinal cord sections were rapidly harvested and frozen on dry ice. Samples were quickly micro-dissected and homogenized using a hand-held pestle with ice-cold SDS sample lysis buffer (Roche Diagnostics, Basel, Switzerland; $10 \mathrm{ml} / \mathrm{mg}$ tissue), containing a cocktail of proteinase inhibitors. The homogenates were centrifuged at $10,000 \times \mathrm{g}$ for $10 \mathrm{~min}$ at $4^{\circ} \mathrm{C}$. Subsequent to the protein concentration being measured using a bicinchoninic acid protein assay kit (cat. no. 23225; Pierce; Thermo Fisher Scientific, Inc.), the homegenates were heated at $100^{\circ} \mathrm{C}$ for $5 \mathrm{~min}$. Proteins $(50 \mu \mathrm{g})$ were separated using SDS-PAGE on a $10 \%$ gel with standard Laemmli solutions (Bio-Rad Laboratories, Hercules, CA, USA) and subsequently transferred onto a polyvinylidene difluoride membrane (Immobilon-P, EMD Millipore). The membranes were washed with Tris-buffered saline with $0.02 \%$ Tween (TBST) and blocked with $5 \%$ non-fat dry milk for $1 \mathrm{~h}$ at room temperature, and incubated overnight with gentle agitation with rabbit anti-JNK (cat. no. sc-7345; 1:1,000; Santa-Cruz Biotechnology, Inc.) and anti- $\beta$-actin (cat. no. A0483; 1:1,000; Sigma-Aldrich; Merck $\mathrm{KGaA}$ ) primary antibodies. Subsequently, membranes were incubated overnight at $4^{\circ} \mathrm{C}$ with a goat anti-rabbit horseradish peroxidase-conjugated secondary antibody (cat. no. RPN4301; 1:10,000; GE Healthcare, Chicago, IL, USA). Between each of these steps, the membranes were rinsed with TBST. All reactions were detected through the enhanced chemiluminescence (ECL) detection method (GE Healthcare). The densities of protein blots were analyzed using Labworks Software (version 4.5; UVP, Inc., Upland CA, USA) and normalized against the values of $\beta$-actin.

Statistical analysis. After the images were captured, the optical density of the same areas of the superficial dorsal horn (lamina I and II) of the five spinal sections were calculated. All data are presented as mean \pm standard error and were analyzed by one-way analysis of variance. Multiple comparisons between the groups were performed using the Student-Newman-Keuls method. All statistical analyses were performed using SPSS version 18.0 software (SPSS Inc., Chicago, IL, USA). $\mathrm{P}<0.05$ was considered to indicate a statistically significant difference.

\section{Results}

Upregulated HDAC2 expression is associated with suppressed MOR activity in the thoracic spinal dorsal horn after $C P$ induction by TNBS. The rats were sacrificed at different time
Table I. Primers sequence for the rat genes characterized in this experiment.

\begin{tabular}{lc}
\hline Gene & \multicolumn{1}{c}{ Sequence } \\
\hline TNF- $\alpha$ & F: 5'-TGATCGGTCCCAACAAGGA-3' \\
& R: 5'-TGCTTGGTGGTTTGCTACGA-3' \\
IL-1 $\beta$ & F: 5'-TGCTGATGTACCAGTTGGGG-3' \\
& R: 5'-CTCCATGAGCTTTGTACAAG-3' \\
IL-6 & F: 5'-GCCCTTCAGGAACAGCTATG-3' \\
& R: 5'-CAGAATTGCCATTGCACAAC-3 \\
GAPDH & F: 5'-CCCCCAATGTATCCGTTGTG-3' \\
& R:5'-TAGCCCAGGATGCCCTTTAGT-3'
\end{tabular}

F, forward; R, reverse; TNF- $\alpha$, tumor necrosis factor- $\alpha$; IL, interleukin.

points to investigate the expression changes of HDAC2 and MOR. Double immunofluorescent staining was performed to reveal the association between HDAC2 and MOR in the thoracic spinal cord. Colocalization of HDAC 2 and MOR were observed both in the control (Fig. 1A-C) and TNBS-treated (Fig. 1D-H) groups. In the TNBS-treated group, HDAC2 expression levels were significantly elevated and persistent from 1 to 5 weeks. Suppressed MOR activity was simultaneously observed and maintained at a low level. Immunostaining density statistical analysis was used to confirm these changes $(\mathrm{P}<0.05$ vs. control group; Fig. 2).

The cellular localization of HDAC2 in the thoracic spinal dorsal horn 5 weeks after CP induction was observed (Fig. 3). Double immunofluorescent staining of HDAC2 with the astrocytic specific marker GFAP (Fig. 3A-C), the microglial marker OX42 (Fig. 3E-G) and the neuronal marker NeuN (Fig. 3I-K) was conducted. The results revealed a clear colocalization between HDAC2 and NeuN (Fig. $3 \mathrm{~K}$ and L); however, HDAC2 rarely colocalized with GFAP or OX42 (Fig. 3D and H).

Intrathecal infusion of HDACi significantly attenuates $C P$-induced mechanical allodynia. To assess whether upregulated HDAC2 contributes to CP-induced pain, AR-42 was used to selectively suppress HDAC2, and thus investigate the nociceptive behavioral consequences and cellular and molecular alterations. The results demonstrated that AR-42 significantly attenuated mechanical allodynia $(\mathrm{P}<0.05$ vs. TNBS; Fig. 4A). However, AR-42 exerted no influence on the response frequency $(\mathrm{RF})$ of sham controlled rats $(\mathrm{P}<0.05$ vs. sham; Fig. 4A). Different stimulations on RFs were tested, and it was observed that RFs of rats were significantly reduced after AR-42 treatment compared with non-treated CP model rats (Fig. 4B). Furthermore, rats treated with only AR-42 did not exhibit differences in RF compared with sham rats. Therefore, AR-42 may attenuate CP-induced chronic pain without interfering with the basic pain behavior.

HDAC2 facilitates the release of the pro-inflammatory cytokines IL1- $\beta$, IL- 6 and TNF- $\alpha$. Our previous study demonstrated that pro-inflammatory cytokines such as IL-1 $\beta$ IL-6, and TNF- $\alpha$ in the spinal dorsal horn increase the status of 

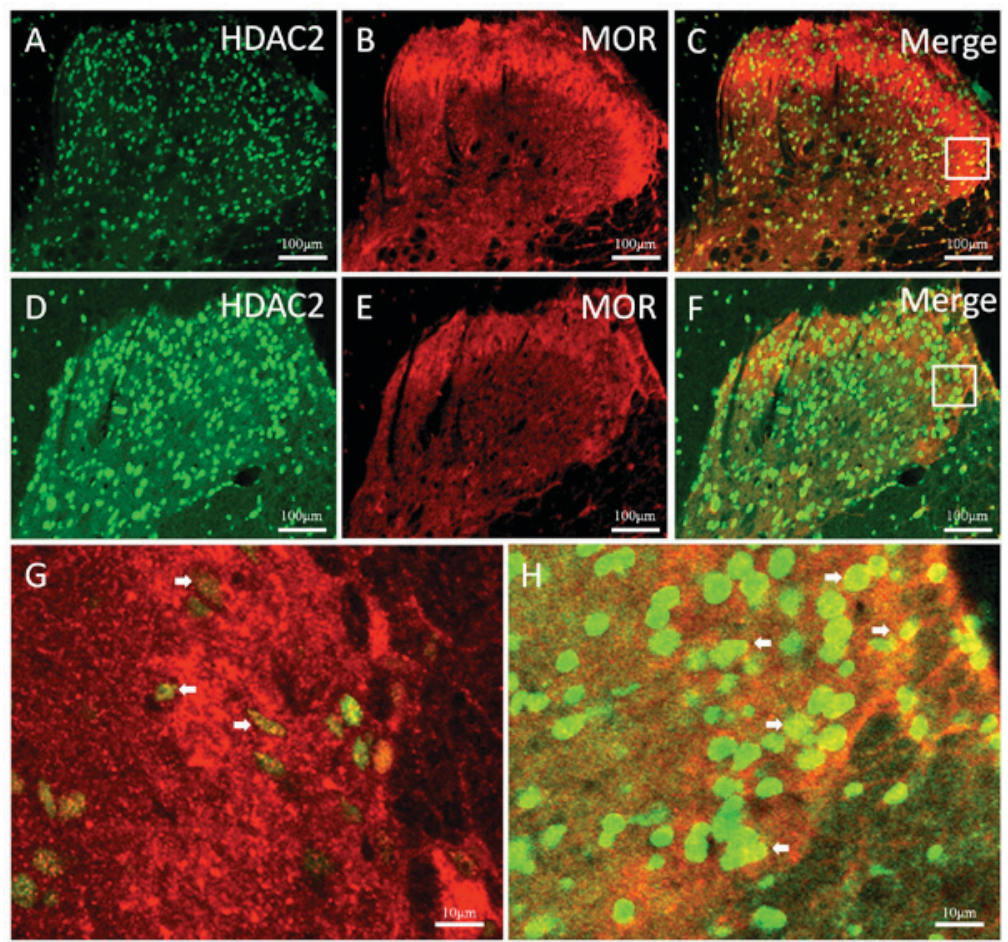

Figure 1. Immunofluorescent double staining for HDAC2 and MOR in the thoracic spinal dorsal horn of CP-induced rats. Immunoreactivities of (A) HDAC2 (green), (B) MOR (red) and (C) merge in the control group. Immunoreactivities of (D) HDAC2, (E) MOR and (F) merge in the CP group. (G) and (H) represent magnified images of the rectangles indicated in the above panels. White arrows indicate areas of merged overlap of HDAC2 and MOR. HDAC2, histone deacetylase 2; MOR, $\mu$-opiod receptor; $\mathrm{CP}$, chronic pancreatitis.

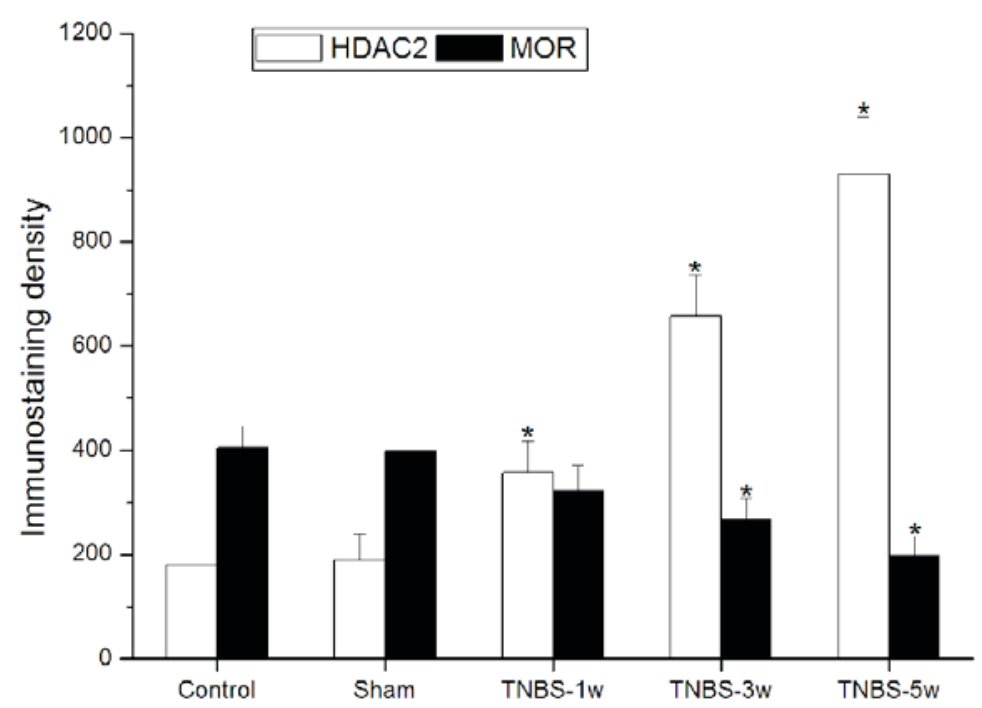

Figure 2. Immunostaining density of HDAC2 and MOR. Data are expressed as the mean \pm standard error. $\mathrm{P}<0.05$ vs. control. HDAC2, histone deacetylase 2 ; MOR, $\mu$-opiod receptor; TNBS, trinitrobenzene sulfonic acid; w, week.

CP (18). Using RT-qPCR, it was observed that the mRNA expression levels of these cytokines were significantly reduced by AR-42 at 3 and 5 weeks in CP rats $(\mathrm{P}<0.05$ vs. TNBS control; Fig. 5), though this effect was not completely reversed $(\mathrm{P}<0.05$ vs. sham; Fig. 5). In addition, AR-42 administration did not significantly affect the sham group $(\mathrm{P}<0.05$ vs. sham control; Fig. 5). These data indicated that HDAC2 may promote the release of pro-inflammatory mediators under chronic $\mathrm{CP}$ allodynia.
Effects of AR-42 on JNK signaling pathways in CP allodynia. As MOR expression is mainly negatively regulated by the JNK signaling pathway (17), the present study selected to further detected the expression levels of this intracellular kinase. Western blotting data indicated that JNK has two subtypes, JNK1 and JNK2, and that the protein expression levels of both were significantly increased after TNBS infusion ( $\mathrm{P}<0.05$ vs. control; Fig. $6 \mathrm{~A}$ and B). However, HDACi AR-42 administration reversed the overexpression of $\mathrm{JNK} 1 / 2$ in $\mathrm{CP}$ 

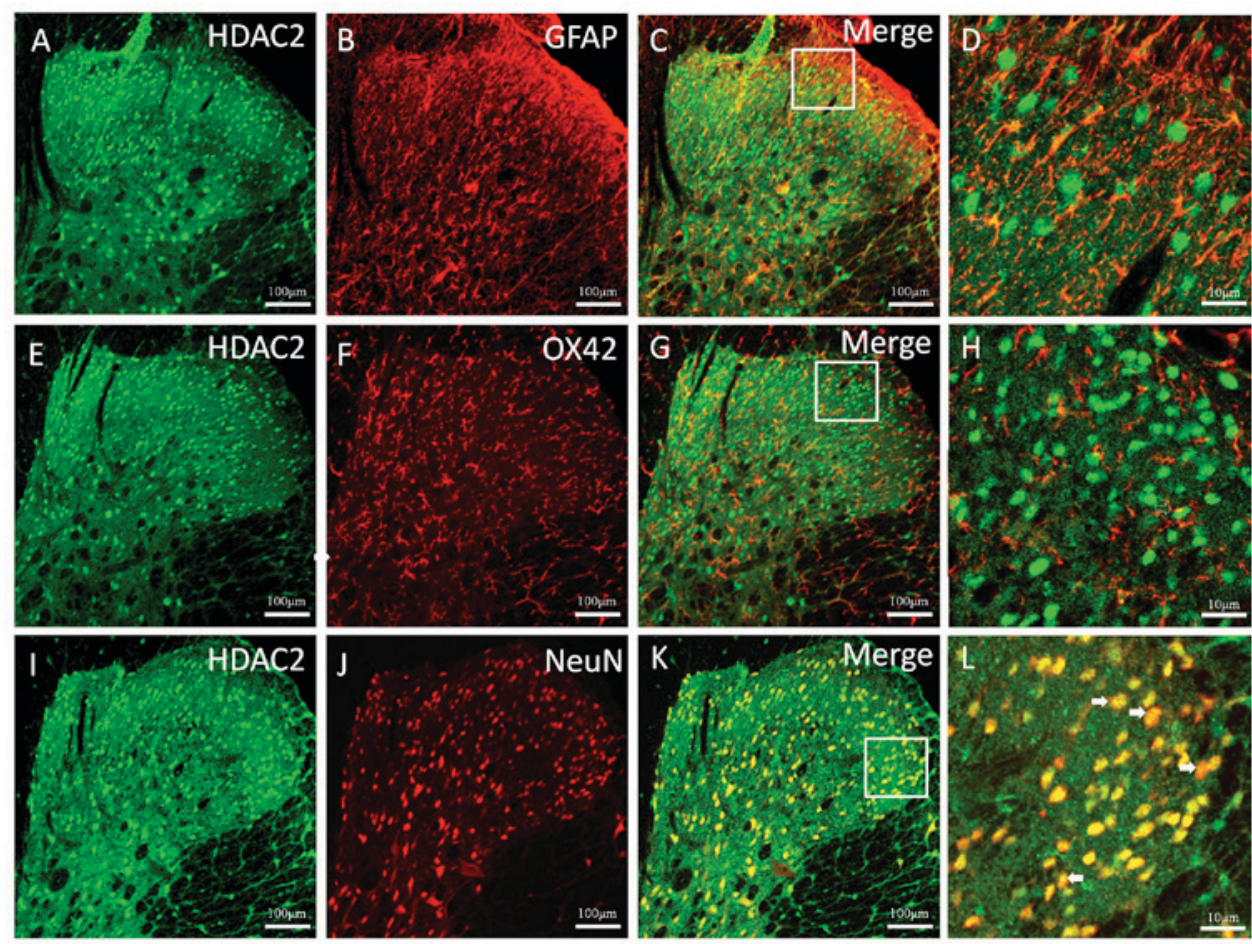

Figure 3. Double immunostaining of the cellular location of HDAC2. (A) HDAC2 and (B) GFAP immunoreactivity, and (C) their merge; (D) represents a magnified image of the rectangles indicated in (C). (E) HDAC2 and (F) OX42 immunoreactivity, and $(\mathrm{G})$ their merge; (H) represents a magnified image of the rectangles indicated in (G). (I) HDAC2 and (J) NeuN immunoreactivity, and (K) their merge; (L) represents a magnified image of the rectangles indicated in (K). White arrows indicate areas of merged overlap NeuN and HDAC2. GFAP, glial fibrillary acidic protein; NeuN, neuronal-specific nuclear protein; OX42, integrin $\alpha-\mathrm{M}$; HDAC2, histone deacetylase 2.

rats $(\mathrm{P}<0.05$, vs. TNBS; Fig. 6A-B), although the expression level remained higher than the control and sham groups. As presented in Fig. 6C, following a TNBS-induced reduction in MOR immunostaining density, HDACi AR-42 significantly rescued MOR activity in CP rats. No significant differences were observed between the control and sham group. In addition, no significant differences were observed on basic MOR expression levels following AR-42 treatment (Fig. 6C). These results suggested that HDAC2 may suppress MOR activity via regulating JNK signaling pathways.

\section{Discussion}

The present study demonstrated that HDAC2 was upregulated and MOR activity was reduced in the spinal cord after $\mathrm{CP}$ induction, and HDAC2 was primarily upregulated in neurons rather than in glial cells in the spinal dorsal horn. As MOR is mainly expressed in neurons (16), it was hypothesized that the contrary expression between HDAC2 and MOR during $\mathrm{CP}$ might be involved in CP-induced allodynia. In the present study, it was demonstrated that HDAC2 increased in the spinal dorsal horn after CP induction. This was also confirmed by immunostaining analysis. Furthermore, the molecular mechanisms underlying the inflammatory process in the CNS following $\mathrm{CP}$ induction were investigated. Intrathecal injection of AR-42 significantly attenuated $\mathrm{CP}$-induced mechanical allodynia and pro-inflammatory cytokine expression levels, following suppressed JNK expression and rescued MOR activity. Though these effects were not completely reversed, these results somewhat elucidated the underlying mechanisms supporting that HDAC2 serves important roles in suppressing MOR activity, thus inducing endogenous analgesia that is disrupted after $\mathrm{CP}$ induction.

In chronic pain, studies on HDACs and their inhibitors are only just beginning to emerge (21). HDACi have attracted increasing interest in recent years due to their potential usage in enhancing neuroplasticity and synaptic activity (22), and reducing neuronal damage $(23,24)$ and neuroinflammation (25). Previous studies have demonstrated that HDAC inhibitors can alleviate inflammatory pain and attenuate the development of hypersensitivity in models of neuropathic pain. Thus, HDACi could be a valid alternative to traditional therapeutic agents by eliminating the side effect of pain (9). However, a clear demonstration that selective interference with HDAC activity can affect $\mathrm{CP}$-induced chronic pain remains lacking. After the HDAC2 selective inhibitor AR-42 was intrathecally administrated, mechanical allodynia was significantly in TNBS treated rats, though not completely. In addition, it was confirmed that AR- 42 could attenuate $\mathrm{CP}$-induced chronic pain without interfering with basic pain behavior. This suggested the potential role of HDAC2 in CP chronic pain induction.

It has been reported that the overexpression of pro-inflammatory cytokines during $\mathrm{CP}$ induction, such as TNF-, IL-1 $\beta$ and IL-6 may promote the allodynia of CP rats $(18,26)$, and these mediators may induce the activation of JNK and extracellular signal-regulated kinase (ERK) signaling pathways, which serve important roles in the maintenance of chronic pain. IL-1 $\beta$ is the most important inflammatory mediator that causes pancreatic islet dysfunction and destruction, which 

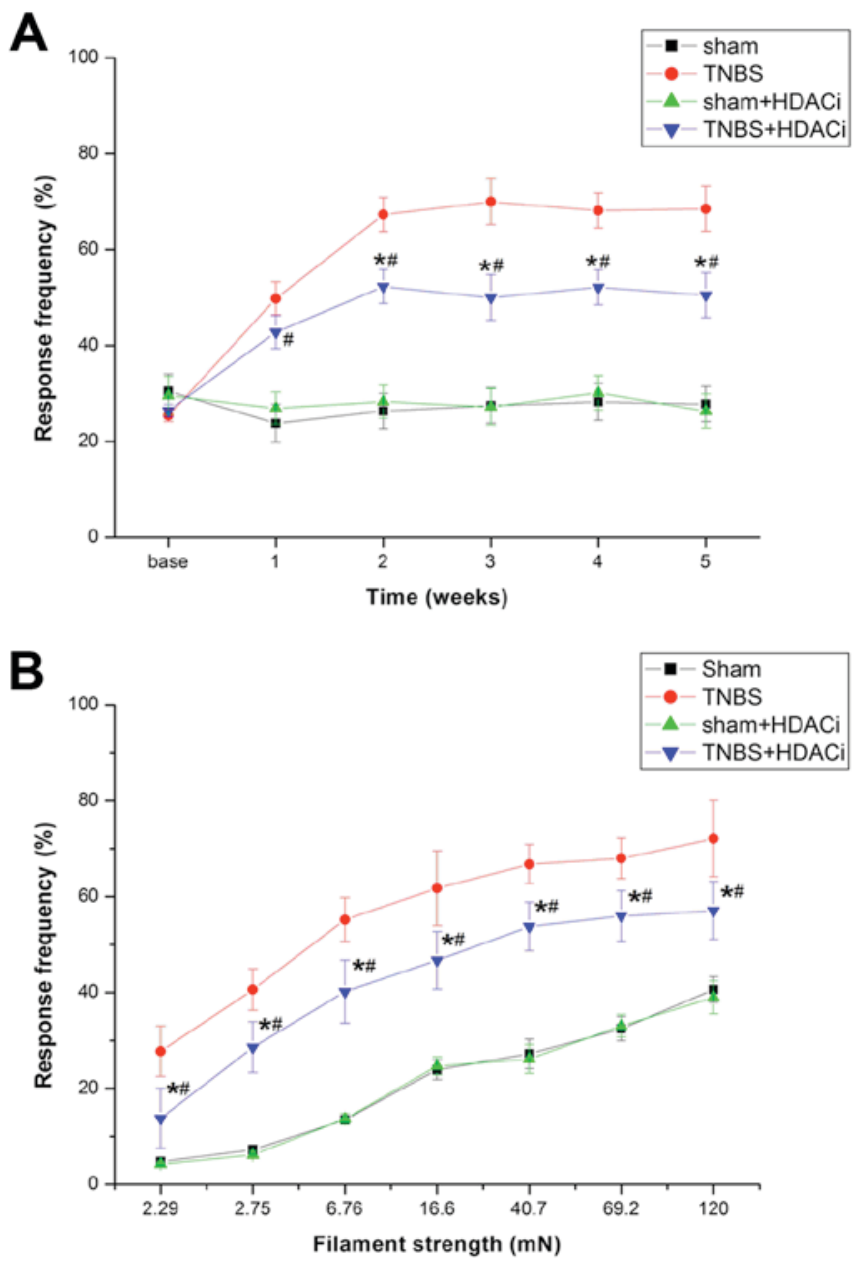

Figure 4. Effects of the HDAC inhibitor AR-42 administration on mechanical allodynia induced by TNBS. Response frequency in relation to (A) time and (B) filament strength. Data are presented as the mean \pm standard error (n=6/group). ${ }^{*} \mathrm{P}<0.05$ vs. TNBS group, ${ }^{\#} \mathrm{P}<0.05$ vs. sham and sham $+\mathrm{HDACi}$ groups. HDAC, histone deacetylase; i, inhibitor; TNBS, trinitrobenzene sulfonic acid.

may promote activation of JNK (27-29); previous studies have also demonstrated positive effect of TNF- $\alpha$ and IL- 6 on JNK activation (30-32). MOR expression is mainly regulated by signaling pathways such as JNK (17). However, ERK exhibits no significantly effect on MOR expression $(33,34)$. Further investigation into the JNK signaling pathway may help reveal the underlying mechanisms involved in CP pain induction. The results of the present study demonstrated that the mRNA expression levels of these cytokines were significantly reduced by AR-42 at 3 and 5 weeks after $\mathrm{CP}$ induction, indicating that HDAC2 may promote the release of pro-inflammatory mediators, maintaining CP allodynia. Though this effect was not completely reversed by AR-42, no significant differences were induced by AR-42 in the sham group. These data are consistent with previous studies that HDACi possesses promising anti-inflammatory activities $(35,36)$.

Western blot analysis demonstrated that JNK expression levels were upregulated after $\mathrm{CP}$ induction, and that inhibition of HDAC2 with AR-42 could significantly reverse JNK1/2 overexpression. Nevertheless, MOR activity was significantly rescued by AR- 42 treatment. Furthermore, AR- 42 did not effect on basic MOR activity. Additionally, the cellular

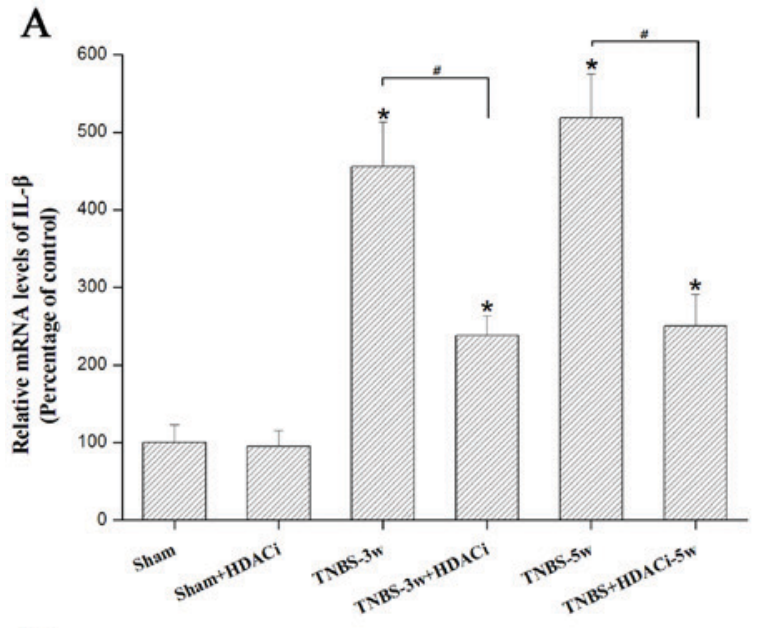

B
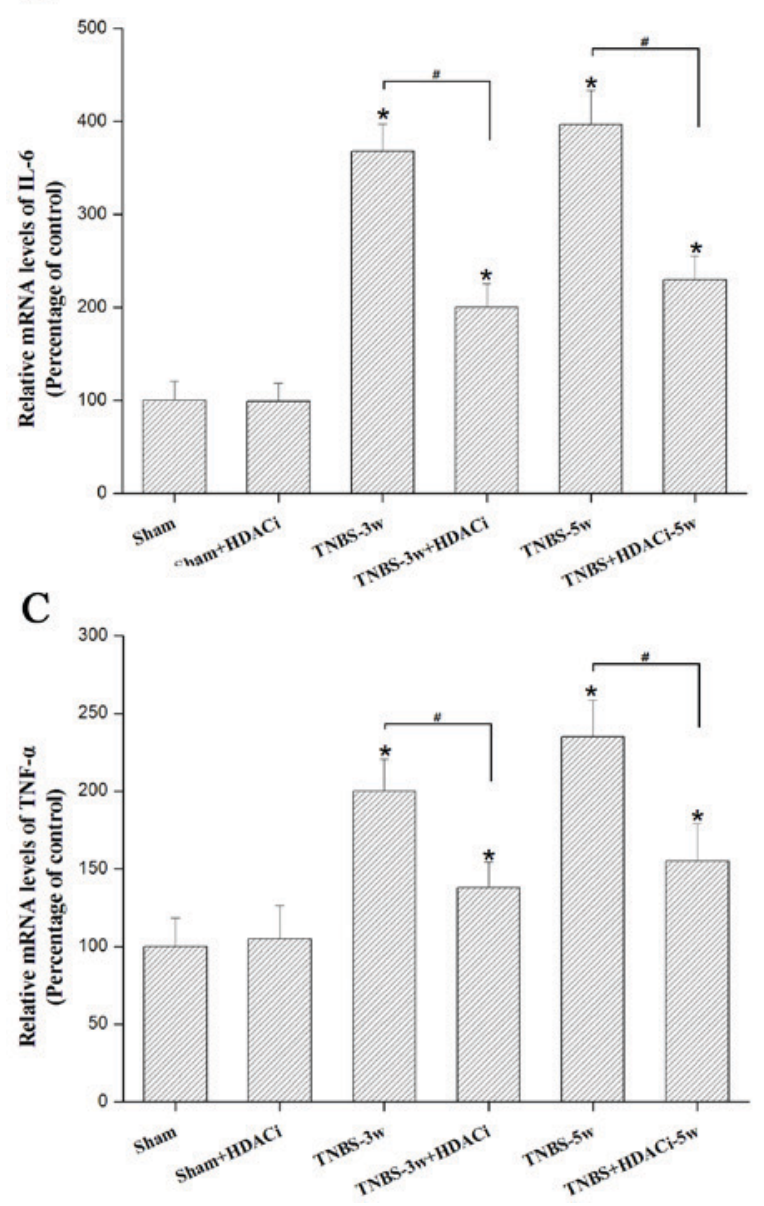

Figure 5. Pro-inflammatory cytokine levels in the spinal dorsal horn following AR-42 treatment. mRNA expression levels of the inflammatory mediators (A) IL-1 $\beta$, (B) IL-6 and (C) TNF- $\alpha$. GAPDH served as an internal reference gene control. Data are presented as the mean \pm standard error. ${ }^{*} \mathrm{P}<0.05$ vs. sham and sham+HDACi groups, ${ }^{\#} \mathrm{P}<0.05$. IL, interleukin; TNF- $\alpha$, tumor necrosis factor- $\alpha$; TNBS, trinitrobenzene sulfonic acid; w, weeks; HDAC, histone deacetylase; i, inhibitor.

localization of HDAC2 was observed in the thoracic spinal dorsal horn, which primarily localized to the cell bodies of neurons and rarely on glial cells, indicating that neuroglia may not directly express HDAC2. These results indicated that HDAC2 may be important in regulating neuroactivity, and is involved in MOR deactivation, causing central sensitization 
A

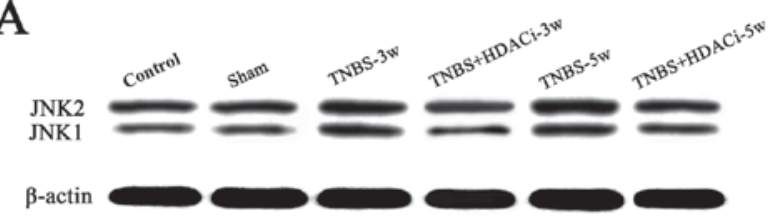

B
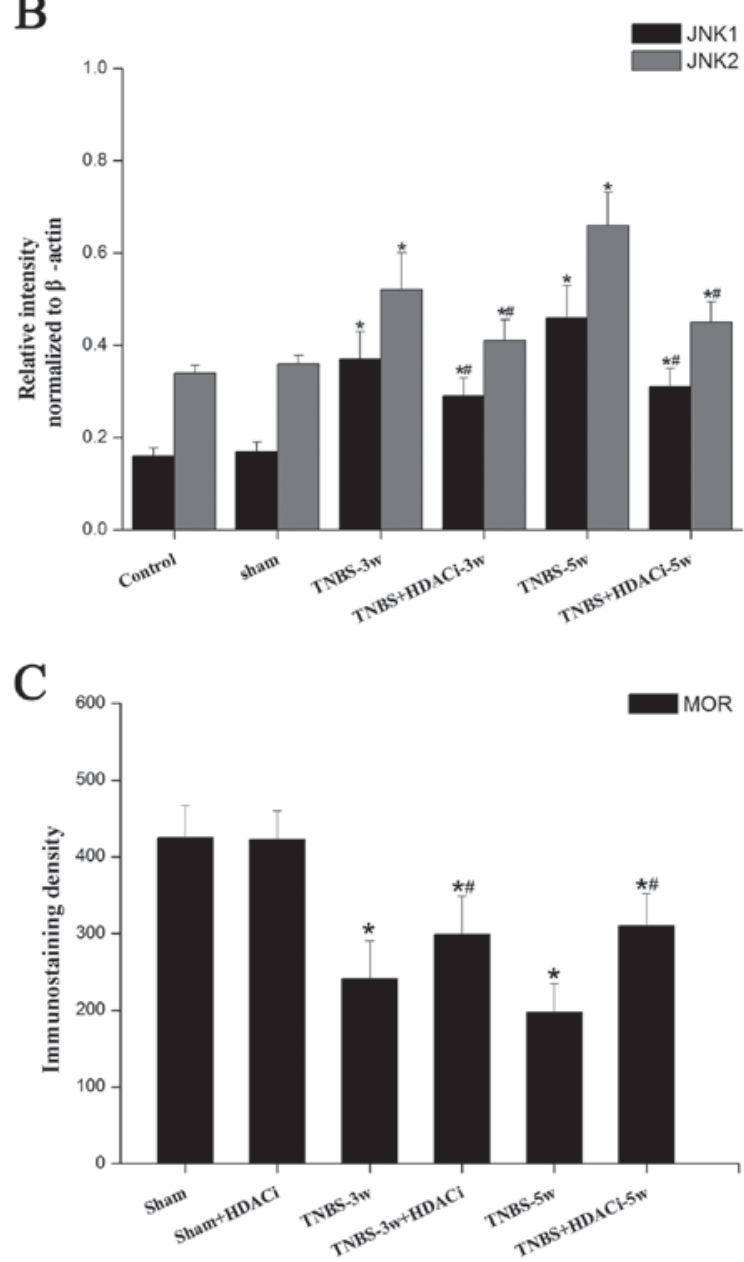

Figure 6. Effects of AR-42 on JNK signaling pathways in chronic pancreatitis. (A) Representative western blot images and (B) quantification of protein expression levels of JNK1/2 in the spinal dorsal horn. (C) Quantification of immunostaining density of MOR following AR-42 treatment. Data are presented as the mean \pm standard error. ${ }^{*} \mathrm{P}<0.05$ vs. control; ${ }^{\#} \mathrm{P}<0.05$ vs. group untreated with AR-42 at the same time point. MOR, $\mu$-pioid receptor; JNK, c-Jun N-terminal kinase; TNBS, trinitrobenzene sulfonic acid; w, weeks; HDAC, histone deacetylase; i, inhibitor.

and persistent pain. The underlying mechanisms may facilitate the release of inflammatory cytokines, thus activating JNK signaling pathways, and finally suppressing MOR activity.

In conclusion, pain induced by CP can be as limiting as the disease itself (37), and requires medical treatment; however, treatment options are limited. Previous studies on humans and animal experimental models have implicated several mechanisms involved in pain, including neuroimmune alterations $(38,39)$. To the best of our knowledge, the present study investigated epigenetic regulation in $\mathrm{CP}$-induced allodynia for the first time, and observed increased HDAC2 activity in the spinal cord and behavioral allodynia after TNBS treatment. Therefore, HDAC2 may be facilitating the release of inflammatory cytokines, activating the JNK signaling pathway, and suppressing MOR activity, causing sensitization and pain induction. These results may facilitate the development of novel therapeutics for patients suffering from $\mathrm{CP}$ pain. Further studies are required to establish drugs with maximum efficacy and fewer side effects.

\section{Acknowledgements}

The present study was supported by the National Natural Science Foundation of China (grant nos. 81400906, 81370928, 81620108008, 31100861 and 2012YQ0302609) and the China Postdoctoral Science Foundation (grant no. 2012M521864).

\section{References}

1. Keefe MD, Wang H, De La O JP, Khan A, Firpo MA and Murtaugh LC: $\beta$-catenin is selectively required for the expansion and regeneration of mature pancreatic acinar cells in mice. Dis Model Mech 5: 503-514, 2012.

2. Braganza JM, Lee SH, McCloy RF and McMahon MJ: Chronic pancreatitis. Lancet 377: 1184-1197, 2011.

3. Olesen SS, Juel J, Graversen C, Kolesnikov Y, Wilder-Smith OH and Drewes AM: Pharmacological pain management in chronic pancreatitis. World J Gastroenterol 19: 7292-7301, 2013.

4. Drewes AM, Krarup AL, Detlefsen S, Malmstrøm ML, Dimcevski G and Funch-Jensen P: Pain in chronic pancreatitis: The role of neuropathic pain mechanisms. Gut 57: 1616-1627, 2008.

5. Feng W, Teng R, Zhao Y, Gao J and Chu H: Epigenetic modulation of Wnt signaling contributes to neuropathic pain in rats. Mol Med Rep 12: 4727-4733, 2015.

6. Gáranton SM: Targeting epigenetic mechanisms for pain relief. Curr Opin Pharmacol 12: 35-41, 2012.

7. Lessans $S$ and Dorsey SG: The role for epigenetic modifications in pain and analgesia response. Nurs Res Pract 2013: 961493, 2013.

8. Gilbert RE, Huang Q, Thai K, Advani SL, Lee K, Yuen DA, Connelly KA and Advani A: Histone deacetylase inhibition attenuates diabetes-associated kidney growth: Potential role for epigenetic modification of the epidermal growth factor receptor. Kidney Int 79: 1312-1321, 2011.

9. Capasso KE, Manners MT, Quershi RA, et al: Effect of histone deacetylase inhibitor JNJ-26481585 in pain. J Mol Neurosci 55: 570-578, 2015.

10. Bai G, Wei D, Zou S, Ren K and Dubner R: Inhibition of class II histone deacetylases in the spinal cord attenuates inflammatory hyperalgesia. Mol Pain 6: 51, 2010.

11. Chiechio S, Copani A,Zammataro M, Battaglia G, Gereau RW IV and Nicoletti F: Transcriptional regulation of type-2 metabotropic glutamate receptors: An epigenetic path to novel treatments for chronic pain. Trends Pharmacol Sci 31: 153-160, 2010.

12. Chiechio S, Zammataro M, Morales ME, Busceti CL, Drago F, Gereau RW IV, Copani A and Nicoletti F: Epigenetic modulation of mGlu2 receptors by histone deacetylase inhibitors in the treatment of inflammatory pain. Mol Pharmacol 75: 1014-1020, 2009.

13. Zhang Z, Cai YQ, Zou F, Bie B and Pan ZZ: Epigenetic suppression of GAD65 expression mediates persistent pain. Nat Med 17: 1448-1455, 2011.

14. Harrison C: Analgesia: Unravelling epigenetic mechanisms of chronic pain. Nat Rev Drug Discov 10: 900, 2011.

15. Jamil MF, Subki MF, Lan TM, Majid MI and Adenan MI: The effect of mitragynine on cAMP formation and mRNA expression of mu-opioid receptors mediated by chronic morphine treatment in SK-N-SH neuroblastoma cell. J Ethnopharmacol 148: 135-143, 2013.

16. Hwang CK, Kim CS, Kim DK, Law PY, Wei LN and Loh HH: Up-regulation of the mu-opioid receptor gene is mediated through chromatin remodeling and transcriptional factors in differentiated neuronal cells. Mol Pharmacol 78: 58-68, 2010.

17. Wagley Y, Hwang CK, Lin HY, Kam AF, Law PY, Loh HH and Wei LN: Inhibition of c-Jun NH2-terminal kinase stimulates mu opioid receptor expression via p38 MAPK-mediated nuclear NF- $\kappa B$ activation in neuronal and non-neuronal cells. Biochim Biophys Acta 1833: 1476-1488, 2013. 
18. Qian NS, Liao YH, Feng QX, Tang Y, Dou KF and Tao KS: Spinal toll like receptor 3 is involved in chronic pancreatitis-induced mechanical allodynia of rat. Mol Pain 7: 15,2011.

19. Ravillah D, Mohammed A, Qian L, Brewer M, Zhang Y, Biddick L, Steele VE and Rao CV: Chemopreventive effects of an HDAC2-selective inhibitor on rat colon carcinogenesis and APCmin/+ mouse intestinal tumorigenesis. J Pharmacol Exp Ther 348: 59-68, 2014.

20. Livak KJ and Schmittgen TD: Analysis of relative gene expression data using real-time quantitative PCR and the 2(-Delta Delta C(T)) Method. Methods 25: 402-408, 2001.

21. Denk F and McMahon SB: Chronic pain: Emerging evidence for the involvement of epigenetics. Neuron 73: 435-444, 2012.

22. Haggarty SJ and Tsai LH: Probing the role of HDACs and mechanisms of chromatin-mediated neuroplasticity. Neurobiol Learn Mem 96: 41-52, 2011.

23. Dietz KC and Casaccia P: HDAC inhibitors and neurodegeneration: At the edge between protection and damage. Pharmacol Res 62: 11-17, 2010.

24. Hashioka S, Klegeris A and McGeer PL: The histone deacetylase inhibitor suberoylanilide hydroxamic acid attenuates human astrocyte neurotoxicity induced by interferon- $\gamma$. J Neuroinflammation 9: 113, 2012.

25. Suh HS, Choi S, Khattar P, Choi N and Lee SC: Histone deacetylase inhibitors suppress the expression of inflammatory and innate immune response genes in human microglia and astrocytes. J Neuroimmune Pharmacol 5: 521-532, 2010.

26. Liu PY, Lu CL, Wang CC, Lee IH, Hsieh JC, Chen CC, Lee HF, Lin HC, Chang FY and Lee SD: Spinal microglia initiate and maintain hyperalgesia in a rat model of chronic pancreatitis. Gastroenterology 142: 165-173.e2, 2012.

27. Cheng H, Xie Z, Jones WP, Wei XT, Liu Z, Wang D, Kulp SK, Wang J, Coss CC, Chen CS, et al: Preclinical pharmacokinetics study of R- and S-enantiomers of the histone deacetylase inhibitor, AR-42 (NSC 731438), in rodents. AAPS J 18: 737-745, 2016

28. Shakespear MR, Halili MA, Irvine KM, Fairlie DP and Sweet MJ: Histone deacetylases as regulators of inflammation and immunity. Trends Immunol 32: 335-343, 2011.

29. Xu Y, Wang D, Zhuang Z, Jin K, Zheng L, Yang Q and Guo K: Hypericin-mediated photodynamic therapy induces apoptosis in K562 human leukemia cells through JNK pathway modulation. Mol Med Rep 12: 6475-6482, 2015.

30. Herrmann JL, Weil BR, Abarbanell AM, Wang Y, Poynter JA, Manukyan MC and Meldrum DR: IL- 6 and TGF- $\alpha$ costimulate mesenchymal stem cell vascular endothelial growth factor production by ERK-, JNK-, and PI3K-mediated mechanisms. Shock 35: 512-516, 2011.
31. Lu ZY, Chen WC, Li YH, Li L, Zhang H, Pang Y, Xiao ZF, Xiao HW and Xiao Y: TNF- $\alpha$ enhances vascular cell adhesion molecule-1 expression in human bone marrow mesenchymal stem cells via the NF- $\kappa \mathrm{B}$, ERK and JNK signaling pathways. Mol Med Rep 14: 643-648, 2016.

32. Liang R, Zhang W and Song YM: Levels of leptin and IL-6 in lungs and blood are associated with the severity of chronic obstructive pulmonary disease in patients and rat models. Mol Med Rep 7: 1470-1476, 2013

33. Kim DK, Hwang CK, Wagley Y, Law PY, Wei LN and Loh HH: p38 mitogen-activated protein kinase and PI3-kinase are involved in up-regulation of mu opioid receptor transcription induced by cycloheximide. J Neurochem 116: 1077-1087, 2011.

34. Bian JM, Wu N, Su RB and Li J: Phosphatidylethanolaminebinding protein is not involved in $\mu$-opioid receptor-mediated regulation of extracellular signal-regulated kinase. Mol Med Rep 11: 3368-3374, 2015.

35. Zhang ZY and Schluesener HJ: HDAC inhibitor MS-275 attenuates the inflammatory reaction in rat experimental autoimmune prostatitis. Prostate 72: 90-99, 2012.

36. Zhang B, West EJ, Van KC, Gurkoff GG, Zhou J, Zhang XM, Kozikowski AP and Lyeth BG: HDAC inhibitor increases histone $\mathrm{H} 3$ acetylation and reduces microglia inflammatory response following traumatic brain injury in rats. Brain Res 1226: 181-191, 2008.

37. Zhang S, Suvannasankha A, Crean CD, White VL, Chen CS and Farag SS: The novel histone deacetylase inhibitor, AR-42, inhibits gp130/Stat3 pathway and induces apoptosis and cell cycle arrest in multiple myeloma cells. Int J Cancer 129: 204-213, 2011.

38. Lucas DM, Alinari L, West DA, Davis ME, Edwards RB, Johnson AJ, Blum KA, Hofmeister CC, Freitas MA, Parthun MR, et al: The novel deacetylase inhibitor AR-42 demonstrates pre-clinical activity in B-cell malignancies in vitro and in vivo. PLoS One 5: e10941, 2010.

39. Ji RR: Neuroimmune interactions in itch: Do chronic itch, chronic pain, and chronic cough share similar mechanisms? Pulm Pharmacol Ther 35: 81-86, 2015.

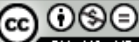

This work is licensed under a Creative Commons Attribution-NonCommercial-NoDerivatives 4.0 International (CC BY-NC-ND 4.0) License. 